\title{
AN UNUSUAL CASE REPORT OF HETEROTROPHIC PREGNANCY
}

Annapurna Hogade ${ }^{1}$, Meenakshi Devarmani², Sneha Krupa ${ }^{3}$.

1. Associate Professor, Department of Obstetrics \& Gynecology, M. R. Medical College, Gulbarga, Karnataka, India

2. Professor, Department of Obstetrics \& Gynecology, M. R. Medical College, Gulbarga, Karnataka, India

3. Post graduate, Department of Obstetrics \& Gynecology, M. R. Medical College, Gulbarga, Karnataka, India

\section{CORRESPONDING AUTHOR:}

Dr. Meenakshi S. Devarmani

M. R. Medical College,

Sedam Road, Gulbarga- 585 105,

Karnataka,India.

E-mail: drssdevarmani@gmail.com

\section{HOW TO CITE THIS ARTICLE:}

Annapurna Hogade, Meenakshi Devarmani,Sneha Krupa. "An unusual Case Report of the Heterotorphic Pregnancy". Journal of Evolution of Medical and Dental Sciences 2013; Vol2, Issue 23, June 10; Page: 40424045.

ABSTRACT: BACKGROUND: Heterotrophic pregnancy is rare complication of pregnancy in which both extra uterine (ectopic pregnancy) and intra uterine pregnancy occur simultaneously. It may also be referred to as combined ectopic pregnancy multiple sited pregnancy or coincident pregnancy. CASE SUMMARY: 26 years old female presented with month of amenorrhea with complaints of pain abdomen since 3 days. She had obstetric score of G4P3L3 with all previous 3 being full term normal vaginal deliveries. USG report was suggestive of ectopic pregnancy and subsequently confirmed with diagnosis of ectopic pregnancy. DISCUSSION: Most of the heterotopic pregnancies present in the first trimester as in this case report. Once a heterotopic pregnancy is diagnosed, the next dilemma that arises is how to manage it without harming the intrauterine pregnancy. Conclusion: Knowledge of the possibility of heterotopic pregnancy and understanding the epidemiological risk factors associated with it are important for early diagnosis and avoiding complications.

KEY WORDS: ectopic, pregnancy, heterotrophic

INTRODUCTION: Heterotopic pregnancy or combined intrauterine and extrauterine pregnancy was first reported in 1708.1 Traditionally, the rate of occurrence has been thought to be 1:30,000 pregnancies, but in the recent literature a rate of 1:2600 has been reported in high-risk groups with the use of assisted reproduction techniques, ${ }^{1}$ although, a rate of 1:100 has been reported with the use of these techniques by Tal et al in $1996 .^{2}$

CASE SUMMARY:A 26 year old female had come with history of 1 month of amenorrhea with complaints of pain abdomen since 3 days on 04/05/2012 at 11:20am. She had a marital life 10 years with obstetric score of G4P3L3 with previous all 3 being full term normal vaginal deliveries. She is moderately built \& nourished with vitals of PR - 108/mins; BP- 110/60 mm Hg. On examination, per abdomen findings - Abdomen was soft, tenderness positive in suprapubic region 
\& right iliac fossa. Per speculum- cervix \& vagina are healthy with no discharge and no bleeding. Per vagina- uterus normal sized, anteverted. Cervical movements tender and culdocentesis is positive. Investigations: Urine pregnancy test- negative. $\mathrm{Hb}-9 \mathrm{gm} / \mathrm{dl}$, total count- 10,000 cells/cu mm. LFT \& RFT- under normal limits. USG report showing ectopic pregnancy with significant fluid in POD \& a small cyst with a solid component in right ovary? Ectopic pregnancy? Rupture ectopic. Emergency laparotomy was done with right salpingectomy \& left tubectomy not done as the patient \& attainder did not give the consent for tubectomy, with intra-op findings right tubal abortion with minimal collection of $500 \mathrm{ml} \&$ products of conception sent for HPR. Confirmed after 10 days as consistent ectopic pregnancy.

Tubectomy was not done as the patient \& attainders were not willing, beta hCG 12,997/microlitre. Final diagnosis- G4P3L3 with right sided tubal abortion. Patient discharged on day 7.

She had come to OPD after 2 months in the month of July with intra-uterine pregnancy of 14 weeks gestation corresponding to the LMP and gestational age previously, when she was admitted \& operated for ectopic pregnancy. Intra-uterine pregnancy was continued with all investigation done \& USG showing 14 weeks of gestational intra-uterine pregnancy with regular ANC.

She finally came on 21/01/2013 with labour pains (final diagnosis would be G4P3L3A with 41 weeks +2 days gestational age with heterotrophic pregnancy in latent phase of labour). She delivered vaginally a live term male baby on $22 / 01 / 2013$ at $6: 35 \mathrm{am}$ of weight $3.5 \mathrm{kgs}$. She underwent tubectomy on $3^{\text {rd }}$ day \& discharged on post operative day 7 .

DISCUSSION: Ectopic pregnancy is a major cause of morbidity and mortality in reproductive age women. There is no single non-invasive test to detect the presence of an ectopic pregnancy. The old traditional teaching that the presence of an intrauterine pregnancy excludes an ectopic is not applicable any more in current practice.

A uterine pregnancy in conjunction with an extrauterine pregnancy is termed as heterotrophic pregnancy. In the past, incidence was 1 in 30,000 pregnancies. As a result of assisted reproductive technology, rate has sky rocketed to 1 in 100 pregnancies. ${ }^{3}$

Mechanisms that have been proposed to explain this includes hydrostatic forced delivering the embryos into corneal or tubal area, the tip of the catheter directing transfer towards the tubal ostia or reflux of uterine secretion leading to retrograde tubal implantation. When a tubal pregnancy coexists with uterine pregnancy, potassium chloride can be injected into the tubal pregnancy.

The false negativity of urine pregnancy test can occur when testing is done too early. Quantitative blood tests and the most sensitive urine test usually begin to detect hCG shortly after transplantation, which can occur anywhere from 6-12 days after ovulation. hCG levels continue to rise through the first 20 weeks of pregnancy, so the chances of false reading decreases percentage to $1.2 \%$ but higher when done early. 4,5 The identification of a live embryo within a gestational sac outside the uterus is the gold standard for the sonographic diagnosis of ectopic pregnancy. ${ }^{5}$

Reece et al regard the common presenting signs and symptoms for heterotopic pregnancy as abdominal pain, adnexal mass, peritoneal irritation, and an enlarged uterus. These presentations are, however, nonspecific and may be confused with other normal or abnormal pregnancy manifestations. ${ }^{6}$

A possible pregnancy must be considered in any woman has amenorrhea with abdominal pain or abnormal vaginal bleeding. A heterotrophic pregnancy may have similar signs and 
symptoms as normal intrauterine pregnancy and ruptured ovarian cyst, corpus luteum or appendicitis. ${ }^{7}$ Blood test and ultrasound can be used to differentiate this condition.

Commonly, hysterectomy with laparotomy was the one and only historical therapeutic option of ruptured cornual pregnancy. Early diagnosis prior to the occurrence of a rupture improves the prognosis and avoids life-threatening complications. The ectopic pregnancy is treated surgically if the intrauterine pregnancy is desired. When the ectopic pregnancy is removed, the intrauterine pregnancy continues in most patients.

A possible pregnancy must be considered in any woman who has amenorrhea with abdominal pain or abnormal vaginal bleeding. A heterotrophic pregnancy may have similar signs and symptoms such as normal intra-uterine pregnancy and ruptured ovarian cyst, corpus luteum or appendicitis. Blood tests and ultrasonography can be used to differentiate these conditions.

There is significant increase in the incidence of heterotrophic pregnancy in patients undergoing ovulation induction. Even greater incidence of heterotrophic pregnancy is reported in pregnancies following assisted reproductive technologies such as in vitro fertilization and gamete intrafallopian transfer.

The prognosis for the extrauterine fetus is very poor, having an estimated $90-95 \%$ mortality rate. The mortality rate for the extrauterine pregnancy is approximately $35 \%$. $^{\mathbf{3}, \mathbf{8}}$

Most of the heterotopic pregnancies present in the first trimester as in this case report. Once a heterotopic pregnancy is diagnosed, the next dilemma that arises is how to manage it without harming the intrauterine pregnancy.

CONCLUSION: All patients who undergo assisted reproduction should be submitted to careful monitoring in early pregnancy. Until recently, especially with the advent of assisted reproductive procedures, it is definitely a fatal condition that might even lead to maternal morbidity and even mortality if there is a delay in intervention assuming it to be an intrauterine pregnancy (IUP). IUP diagnosed with ease in this case will enumerate how the source of haemoperitoneum was least thought to be of tubal origin because of the rarity in the incidence of heterotopic pregnancy.

\section{REFERENCES:}

1. Bright DA, Gaupp FB. Heterotopic pregnancy: A reevaluation. J Am Board Fam Pract 1990; 3 (2): $125-8$.

2. Po-Jen Cheng, Ho-Yen Chueh and Jian-Tai Qiu. Heterotopic Pregnancy in a Natural Conception Cycle Presenting as Hematometra; OBSTETRICS \& GYNECOLOGY 2004; 104; 5(2):1195-1198.

3. Bello GV, Sehonols D, Moshirapus J. Combined pregnancy: The Mount Sinai experience; Obstet Gynecol Surv 1986;46:603.

4. Reece EA, Petrie RH, Sirmans MF, Finster M, Todd WD. Combined intrauterine and extrauterine gestations: a review. Am J Obstet Gynecol 1983; 146:323-30.

5. Richard SR, Stempel LE, Carton BD. Heterotrophic pregnancy; Reappraisal of incidence; Am J Obstet Gynecol 1982; 142:928.

6. Stabile I, Grudzinskas JG. Progress in Obstetrics and Gynaecology: Ectopic pregnancy; what's new? 11 ed. Edinburgh: Churchill Livingston; 1994. p. 281-309. 
7. Erdogan NOHUZ et al. Ruptured cornual heterotopic pregnancy: a possible cause of acute abdomen after voluntary pregnancy termination; Anatol J Obstet Gynecol 2011; 1:2.

8. Rawal S et al. Heterotopic pregnancy with spontaneous intrauterine conception: A rare clinical entity with diagnostic dilemma; Kathmandu University Medical Journal 2008;6;1(21):105-108

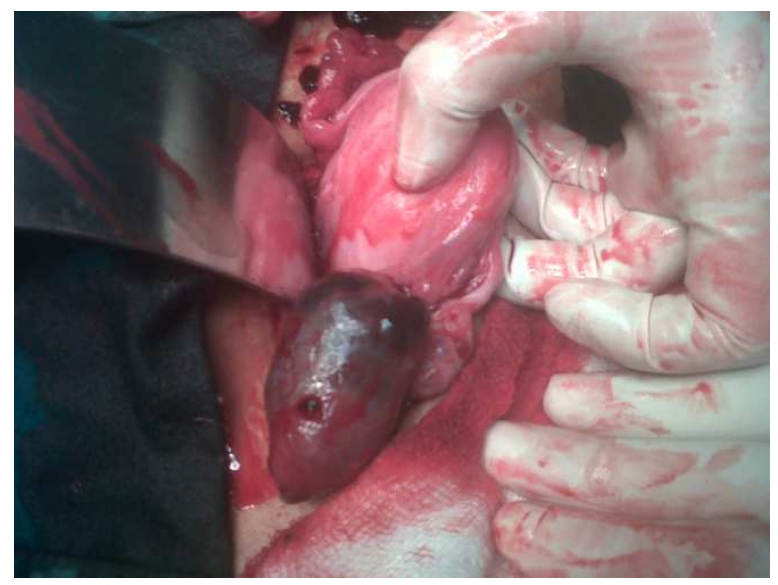

"Features showing right sided tubal abortion". 